\title{
Simultaneous time and size perception
}

\author{
EWART A. C. THOMAS and NANCY E. CANTOR \\ Stanford University, Stanford, California 94305
}

\begin{abstract}
Judgments of the apparent duration and size of visually presented circles vary directly with the duration and size of the presented stimuli. When the frequencies of stimulus duration (short vs. long) and stimulus area (small vs. large) are varied, perceived size and duration are directly related to the frequency of the lower attribute value (short or small). These data are compared to the predictions of different information-processing models. The model which accounts for the data best is one in which it is assumed that perceived size and perceived duration grow together over the course of time spent sampling size information, and that attribute frequency affects the rate of sampling and/or the point at which sampling stops.
\end{abstract}

In an attempt to explain the role of nontemporal stimulus information in the estimation of brief stimulus durations $(<100 \mathrm{msec})$, the suggestion has been made that estimates of duration are affected by the time spent processing nontemporal information (Avant, Lyman, \& Antes, 1975; Thomas \& Weaver, 1975). The validity of this suggestion has been explored in a recent study by Thomas and Cantor (1975) in which subjects were presented with solid circles varying in size (nontemporal information) and in duration, and were asked to judge the size and the duration of the stimuli. This study shows that perceived duration is directly related to stimulus size, and perceived size is directly related to stimulus duration. A model was proposed for the processing of size information, which accounts for both sets of judgments reasonably well. What these data support is not merely a processing model, but the model plus the assumption that processing time is directly related to perceived duration.

Having judgments of both stimulus attributes, duration and size, allows one to investigate the effect of size on perceived duration and the dual effect of duration on perceived size. This, in turn, leads to stronger tests of the present approach than would be possible if only duration estimates were available. Further tests of the approach are possible when the range of tasks is extended and when a variety of processing models are examined. This is the tack pursued in the present study. In the Thomas and Cantor (1975) study, the subject's perception of one stimulus attribute was related to the objective values of the other attribute; for example, perceived duration was related to stimulus size. In the present study, the design allows, in addition, perceived duration to be related to perceived size of a given stimulus, as the frequencies of stimulus attributes are varied. Variation in attribute frequency has been

This research was supported by Grant No. GB-43175 from the National Science Foundation to the first author. We wish to thank Gordon H. Bower, Barbara Sakitt, and the referees for helpful comments. employed very recently by Mo (1975) in a study of the effects of numerosity on temporal judgments.

To fix ideas, suppose that a stimulus consists of a circle, which has size $A_{1}$ or $A_{2}\left(>A_{1}\right)$, presented for a duration $T_{1}$ or $\left.T_{2} \otimes T_{1}\right)$. In a block of trials, $A_{1}$ will have a certain frequency, $\mathrm{Pa}_{a}$ say (a for "area"), and $\mathrm{A}_{2}$ a frequency $1-\mathrm{p}_{\mathrm{a}}$; and $\mathrm{T}_{1}$ will have a frequency, $\mathrm{p}_{\mathrm{t}}$ say ( $t$ for "time"), and $T_{2}$ a frequency $1-p_{t}$. For such a block, the subject's task is either (a) to judge the duration of each stimulus immediately after it is presented on a rating scale, $0=$ "short," $1=$ "medium," $2=$ "long," and to estimate the $A_{1}$ frequency at the end of the block, or (b) to judge the size of each stimulus immediately after it is presented $(0=$ "small," $1=$ "medium," 2 = "large"), and to estimate the $T_{1}$ frequency at the end of the block. Task a yields estimates of perceived duration, $\tau$ (the average numerical response), and, similarly, task $b$ yields estimates of perceived size, $\alpha$. The judgments required at the end of a block presumably force the subject to process information about both stimulus attributes on each trial. For each type of judgment, that is, for $\tau$ and $\alpha, \mathrm{p}_{\mathrm{a}}$ is varied across blocks, keeping $\mathrm{p}_{t}=.5$, and $\mathrm{p}_{t}$ is varied across other blocks, keeping $p_{a}=.5$. Therefore, the data for each of the four stimuli $\left(A_{\mathbf{i}}, T_{\mathbf{j}}\right), i, j=1,2$, consist of the functions relating $\tau$ to $\mathrm{p}_{\mathrm{a}}$ and to $\mathrm{p}_{\mathrm{t}}$, and relating $\alpha$ to $p_{a}$ and to $p_{t}$. Clearly, for each stimulus, one can use these data to relate $\tau$ to $\alpha$ as $\left(\mathrm{p}_{\mathrm{a}}, \mathrm{p}_{\mathrm{t}}\right)$ is varied across blocks. Also, for each setting of $\left(\mathrm{p}_{\mathrm{a}}, \mathrm{p}_{\mathrm{t}}\right), \tau$ and $\alpha$ can be related to $A_{i}$ and $T_{j}$.

The use of a 3-point rating scale to measure perceived duration and size is a more direct method than, say, a paired-comparison procedure. The averaging of the numerical responses to obtain the best estimate of perceived duration is the simplest way of summarizing the data and can be justified in at least three ways: (i) Subjects can be trained to associate response $k(=0,1$, or 2$)$ with stimulus duration $T^{\prime}+k \Delta$, where, for example, $\mathrm{T}^{\prime}$ and $\Delta$ might equal 20 and $40 \mathrm{msec}$, respectively. If we make the simplifying assumption that, as a result of training, perceived duration takes on only three values, 
associated with stimulus durations of $T^{\prime}, T^{\prime}+\Delta$, and $T^{\prime}$ $+2 \Delta$, then the rating scale can be regarded as an interval scale and averaging is justified. (ii) Other methods of analyzing the ratings rest on assumptions that are at least as restrictive as those in (i). [See Thomas and Weaver (1975), Footnote 3, for an application of the theory of signal detectability.] (iii) In the Thomas and Weaver (1975) study, it was found that the difference, d, between the perceived durations of two "empty" visual fields, when transformed into real time by $t=T^{\prime}+d \Delta$, was nearly equal to the difference in actual durations, although the perceived duration of each field was an underestimate. This suggests that the rating scale gives numbers that are linearly related to real time, and provides further justification for regarding the rating scale as an interval scale. Similar arguments can be used to justify the averaging of size ratings.

It might be supposed that the direct relation observed between $\tau$ and $\mathrm{A}_{\mathbf{i}}$ and between $\alpha$ and $\mathrm{T}_{\mathbf{j}}$ reflect the mutual contribution to apparent brightness of duration and area (Ricco's law), and that, therefore, the phenomenon resides in the peripheral visual system. There are three reasons why we have preferred not to take this view. First, in our experiments, the stimuli consist of solid, red circles on a well lit, white background so that it is unlikely that the larger circle appears brighter than the smaller one. Second, in a study using intensity, not area, as the nontemporal information, Allan, Kristofferson, and Wiens (1971) found that, for durations for which Bloch's law holds, temporal judgments appear to be based on duration information rather than on apparent brightness. Third, models that view the phenomenon as peripheral make no predictions about the effects of varying attribute frequencies $\left(\mathrm{p}_{a}\right.$ and $\left.\mathrm{p}_{\mathrm{t}}\right)$.

If we assume that $\tau$ is a weighted average of some monotonic function of $T_{j}$ and of the time spent processing information about size (Thomas \& Cantor, 1975, Equation 1), we can investigate the dependence of $\tau$ and $\alpha$ on $\mathrm{p}_{\mathrm{a}}$ and $\mathrm{p}_{\mathrm{t}}$ that would be predicted by various models for size estimation. These models need to contain assumptions about the magnitude $(\alpha)$ of the size estimate and about the processing time required to compute this estimate. We now consider some alternatives.

For ease of exposition, we assume that there are only two response alternatives, 0 and 1 (instead of 0,1 , and 2 as in the present experiments). This allows us to equate $\alpha$ and $\tau$ with the probability of responding " 1 ," but our conclusions about $\alpha$ and $\tau$ do generalize to the case of three response alternatives. Concerning the effects of frequency on response magnitude, one might suppose either of the following: (1) The size information on each trial is a random variable, $\mathrm{X}$, the median of which equals the objective value of the attribute $\left(A_{1}\right.$ or $\left.A_{2}\right)$. The subject has a decision criterion, $c$, depending on $p_{\mathbf{a}}$, such that the subject responds "large" if and only if $X>c$. As is done in the theory of signal detectability (Green \& Swets. 1966), we can suppose that $c$ is an increasing function of $\mathrm{p}_{\mathrm{a}}=\mathrm{P}\left(\mathrm{A}_{1}\right)$. This would imply that, for both $A_{1}$ and $A_{2}, \alpha$ decreases as $p_{a}$ increases. If a similar model is applied to temporal judgments, the prediction would be that $\tau$ decreases as $p_{t}$ increases. (2) The subject establishes a subjective standard, $c^{*}$, which is close to the average of the presented sizes $A_{1}$ and $A_{2} . c^{*}$ is thus the adaptation level defined by Helson, 1947), and it decreases as $p_{a}$ increases. The subjective magnitude of $A_{1}$ is assumed to be obtained by comparing $A_{1}$ with the larger standard, $c^{*}$, and $A_{1}$ appears smaller when the (positive) difference $c^{*}-A_{1}$ is larger. Similarly, the subjective magnitude of $A_{2}$ is greater when the positive difference $\mathbf{A}_{2}-c^{*}$ is greater. This would imply that, for both $\mathbf{A}_{\mathbf{1}}$ and $\mathbf{A}_{\mathbf{2}}, \alpha$ increases as $\mathrm{p}_{\mathrm{a}}$ increases. Applying a similar model to temporal judgments, the prediction would be that $\tau$ increases as $\mathrm{p}_{t}$ increases. Thus, Assumptions 1 and 2 give opposite predictions.

Concerning the effects of frequency on processing time (PT) of size information, one might suppose either of the following: (3) When a variable $X$ is compared with a criterion, $c$ (as in Assumption 1, or $\mathrm{c}^{*}$ as in Assumption 2 above), PT is inversely related to $|\mathrm{X}-\mathrm{c}|$ (Thomas \& Myers, 1972). To simplify matters, we assume that this inverse relationship is linear, so that average PT is linearly related to the average of $|X-c|$. This latter average is a minimum when $c$ equals the median $\mathrm{X}$ value, $\mathrm{A}_{\mathrm{i}}$. Therefore, provided $\mathrm{A}_{1}<\mathrm{c}<\mathrm{A}_{2}$ (in which case performance is above chance level for large and small circles), for small circles, as $c$ increases, the average of $|\mathrm{X}-\mathrm{c}|$ increases and the average PT decreases; for large circles, the opposite is true. This conclusion holds also when $c$ is replaced by $c^{*}$ from Assumption 2. (4) The time to process the information about a stimulus attribute $\left(A_{i}\right)$ is inversely related to the frequency with which $A_{i}$ is presented. Thus, for small circles, average PT decreases as $p_{a}$ increases, and for large circles, the opposite is true.

Let us now consider the (full) model consisting of Assumptions 1 and 3. Since $\tau$ is directly related to PT, and since $c$ increases as $\mathrm{p}_{\mathrm{a}}$ increases, we have from Assumption 3 that, for small circles, $\tau$ decreases as $p_{a}$ increases, and, for large circles, $\tau$ increases as $\mathrm{p}_{\mathrm{a}}$ increases. But from Assumption 1, $\alpha$ varies inversely with $\mathrm{p}_{\mathrm{a}}$. Therefore, as $\mathrm{p}_{\mathrm{a}}$ changes, $\tau$ varies directly with $\alpha$ for small circles, and inversely with $\alpha$ for large circles. The same conclusion is reached by considering the model consisting of Assumptions 1 and 4.

Similarly, considering the model consisting of Assumptions 2 and 3, we see that, for small circles, as $\mathrm{p}_{\mathrm{a}}$ increases, both $\tau$ and $\alpha$ increase; for large circles, as $\mathrm{p}_{\mathrm{a}}$ increases, $\tau$ decreases while $\alpha$ increases. Here, as above, $\tau$ varies directly with $\alpha$ only for small circles. For the model consisting of Assumptions 2 and 4, $\tau$ varies directly with $\alpha$ only for large circles.

These full models also make predictions about the dependence of $\tau$ on $A_{i}$, for fixed $\left(\mathrm{p}_{a}, p_{t}\right)$ and fixed 
$T_{\mathbf{j}}$. To derive these predictions, we need only consider the difference in PT for large and small circles, $\mathbf{P T}_{1}-\mathbf{P T}_{2}$. It is easy to show that these four models all predict that $\mathrm{PT}_{1}-\mathrm{PT}_{2}$ is positive for some values of $\mathrm{pa}_{\mathrm{a}}$ and negative for other values. That is, the models predict the direct relationship between $\tau$ and $A_{i}$, observed by Mo and Michalski (1972) and Thomas and Cantor (1975), only for certain values of $p_{a}$.

To summarize, the four models obtained by combining the above assumptions predict that, as $p_{a}$ varies, (i) $\tau$ is directly related to $\alpha$ for either small or large circles, but not both, and, consequently, (ii) $\tau$ is directly related to $A_{i}$ only for certain values of $p_{a}$. It was stated that Assumptions 1 and 2 lead to relationships between $\tau$ and $p_{t}$, but it is not clear how these and Assumptions 3 and 4 can be used to derive a relationship between $\alpha$ and $\mathrm{p}_{\mathrm{t}}$.

We turn now to the model proposed by Thomas and Cantor (1975) to account for their results on the dependence of $\tau$ on $\mathrm{A}_{\mathrm{i}}$ and $\alpha$ on $\mathrm{T}_{\mathrm{j}}$. This model assumes that the first stage of processing is an unprocessed, internal representation of the presented stimulus, which representation is constant while the stimulus is on and decays after stimulus offset. This representation is "sampled" by a scanner until a criterion is reached (as in randomwalk models of choice reaction time; for example, Laming, 1968; Link \& Heath, 1975), or until the "quality" of the samples falls below a certain level owing to decay of information after offset (as in Thomas, 1973), whichever occurs first. It is further assumed that the representation is spatial in nature and that the scanner takes small, possibly overlapping, spatial samples, one after another, and transfers them to a size comparator where the new information about size contained in each sample is integrated with the size information already there. At any point in time, the output of the size comparator is the amount of the presented circle (roughly speaking, the amount of "red," since the circles in this study are red) accumulated up to that time, and this amount determines the apparent size of the presented circle at that time. Because of sequential sampling, apparent size grows over (processing) time. The rate of growth of apparent size is assumed to be (i) directly related to $A_{i}$, because with a larger circle there is greater chance that early samples are taken from nonoverlapping parts of the circle, giving a greater average amount of new information in each sample, and (ii) greater, at a given point in time, if the stimulus is on than if it is off, because the representation of the stimulus decays after stimulus offset. Processing is assumed to be terminated when the rate of growth of apparent size falls below a certain value. This could be because the stimulus is small relative to the size of the samples taken by the scanner so that complete information about size is obtained after one or two samples and before stimulus offset. This possibility is illustrated in curves $\mathrm{C}(1,30)$ and $\mathrm{C}(2,70)$ of Figure 1 . Or, it could be because the stimulus is relatively large and the duration is relatively short so that the representation decays completely before all the size information is scanned. This is illustrated in curves $\mathrm{C}(3,30), \mathrm{C}(4,30)$, and $\mathrm{C}(4,70)$ of Figure 1. From Figure 1, we can see that there is a variety of possible relationships between stimulus area and duration, on the one hand, and perceived size and duration, on the other.

The properties (for example, slope and curvature) of the curves shown in Figure 1 are consistent with processing models other than the one detailed here, and it is these properties, rather than the details of the model presented, which we regard as necessary in order to account for existing data. For example, it could have been assumed that the scanner extracts only contour information, and that the scanning time is longer for larger circles because the contour (perimeter) is larger. Also, it could have been assumed that "sampling" is not sequential but is simultaneous along a number of independent channels, this number being directly related to the area of the circle. Perceived duration can be assumed to depend partly on the difference between the sampling times of the slowest and fastest channels, and perceived size to depend on the number of channels activated. It may be possible to discriminate among these possibilities, but for the present we focus on the interpretation of Figure 1 detailed above.

Within this model, there are two ways in which variation of attribute frequency can affect $\tau$ and $\alpha$. The first is by affecting the rate of growth, that is, by changing

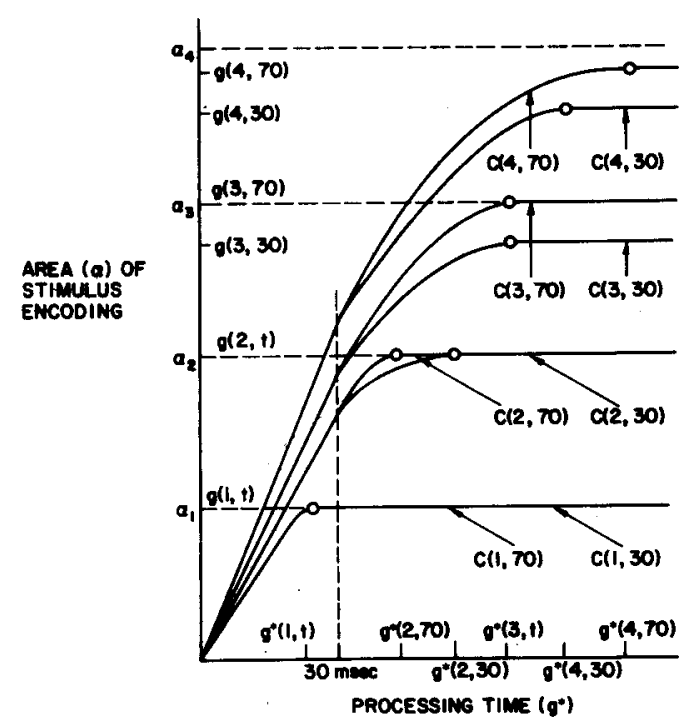

Figure 1. Accumulation of size information, $\alpha$ ( $\equiv$ area of stimulus encoding), over processing time, $g^{*}$, as a function of stimulus area, $A_{i}$, and stimulus duration $t=30$ or 70 msec. $\alpha_{i}$ is the internal representation of stimulus area, with an infinite t. On each curve, $C(I, t)$, the open circle represents the point at which procescing stops. This point determines the asymptotic size of stimulus encoding, $g(I, t)$, and the processing time, $g^{*}(I, t)$. (Taken from Thomas \& Cantor, 1975). 
the curve of apparent size vs. time; and the second is by varying the point at which processing stops, the sizetime curve remaining unchanged. The justification of either type of frequency effect is an open issue, but we might consider the following suggestions.

Considering the possible effects of $\mathrm{p}_{\mathrm{a}}$ on rate, we might suppose that, when there is a preponderance of small circles, sampling is very likely to start in the middle of every circle, that is, close to the imaginary fixation point, rather than at the edges of the circle. In other words, high values of $\mathrm{p}_{\mathrm{a}}$ force the subject into a systematic sequential sampling strategy, whereas at low values of $\mathrm{p}_{\mathrm{a}}$ a random strategy is more likely to be used. This means that early samples are very likely to contain parts of the circle (rather than parts of the background), implying that the rate of growth of apparent size would be higher when $\mathrm{p}_{\mathrm{a}}$ is high than when $\mathrm{p}_{\mathrm{a}}$ is low. Next. when there is a preponderance of short durations, we might assume that the rate at which samples are taken is increased in an attempt to maintain a certain level of acquired information. The result of this would be to have a greater rate of growth when $p_{t}$ is high than when $p_{t}$ is low. Referring to Figure 1, the effect of increasing the rate of growth is to raise the curve, $C(I, t)$, and this would, in general, increase the asymptotic apparent size, $\mathrm{g}(\mathrm{I}, \mathrm{t})$. (The subject's numerical judgment $\alpha$ is assumed to be directly related to g.) The effect of increasing rate on processing time depends on $A_{i}$. For circles larger than a certain value, increased rate of growth leads to increased processing time because it takes longer to reach the point at which the "quality" of samples falls below a certain criterion. inis effect would be observed if we were to "raise" curve C $(4,30)$ of Figure 1 to $\mathrm{C}(4,70)$. For very small circles, increased rate of growth leads to decreased processing time, as when $\mathrm{C}(2,30)$ is "raised" to $\mathrm{C}(2,70)$. Therefore, for relatively "large" circles, both $\tau$ and $\alpha$ would be directly related to $\mathrm{p}_{\mathrm{a}}$ and $\mathrm{p}_{\mathrm{t}}$.

Corsidering the possible effects of $p_{a}$ and $p_{t}$ on the time at which processing stops, we might suppose that processing time is increased for all stimuli when the task as a whole becomes more difficult. This could occur because the subject lowers the "quality" criterion at which sampling stops. Assuming that task difficulty is increased by having a preponderance of small circles or of short durations (this assumption is supported by the comments of subjects in our experiments), we would again predict that both $\tau$ and $\alpha$ are directly related to $\mathrm{p}_{\mathrm{a}}$ and to $\mathrm{p}_{\mathrm{t}}$.

Since the predicted effects of varying $p_{t}$ have been obtained by means of assumptions about how size information is processed, it is of interest to see how variations in $\mathrm{p}_{t}$ affect $\tau$ when "blank" durations (white field with no circle) are used. The following experiment was run to provide data bearing upon the theoretical possibilities discussed above.

\section{METHOD}

Three groups of subjects participated in separate conditions of the experiment.

\section{Group 1: Temporal Judgments}

Subjects. Twenty-one Stanford University undergraduates recejving credit toward an introductory psychology course participated in individual sessions of about $1 \mathrm{~h}$.

Stimuli. The stimuli were red circular areas presented on a lighted background of luminance $91.23 \mathrm{~cd} / \mathrm{m}^{2}(26.64 \mathrm{fL})$ in a modified three-field Iconix tachistoscope. The circular diameters were $8.33 \mathrm{~mm}$ for stimulus $A_{1}$ and $10.32 \mathrm{~mm}$ for stimulus $A_{2}$, subtending visual angles of $.8^{\circ}$ and $1^{\circ}$, respectively. A stimulus was presented for 30 or $70 \mathrm{msec}$ on each trial.

Responses. Each subject was trained to discriminate between blank durations of $15 \mathrm{msec}$ ("short"), $50 \mathrm{msec}$ ("medium"), and $85 \mathrm{msec}$ ("long"), and between $A_{1}$ and $A_{2}$ shown for $a$ constant $50 \mathrm{msec}$. On each trial, subjects were required to categorize the duration of presentation of the circle as "short," "medium," or "long." At the end of each block of 24 trials, subjects gave a judgment of the frequency of presentation of $A_{1}$ and $\mathbf{A}_{2}$. Subjects were given a practice block of eight trials, without feedback, after receiving training in the discrimination tasks and before beginning the experimental blocks.

Procedure. Each session consisted of four experimental blocks, each containing 24 trials. Each trial in all four blocks consisted of a blank warning field of $500 \mathrm{msec}$, followed after a $500-\mathrm{msec}$ delay by a 30 - or $70-\mathrm{msec}$ exposure of the circle. After a further $500-\mathrm{msec}$ delay, a response card was shown for $500 \mathrm{msec}$ in order to elicit the subject's response. The four trial blocks varied according to the frequency of small circles $\left(\mathrm{p}_{\mathrm{a}}\right)$ or of short durations $\left(\mathrm{p}_{\mathrm{t}}\right)$. The following frequency combinations were used: $\left(\mathrm{p}_{\mathrm{a}}, \mathrm{p}_{\mathrm{t}}\right)=(1 / 3,1 / 2),(2 / 3,1 / 2),(1 / 2,1 / 3)$. $(1 / 2,2 / 3)$. The presentation of the trial blocks was counterbalanced over subjects, within the constraint that blocks in which $p_{t}$ was $1 / 2$ were presented consecutively. and those in which $\mathrm{p}_{\mathrm{a}}$ was $1 / 2$ were also presented consecutively. Stimuli within each block were randomly ordered and the order was kept constant over all subjects.

\section{Group 2: Size Judgment}

Subjects. Twenty-one Stanford University undergraduates receiving credit toward "Introductory Psychology" participated in individual $1-h$ sessions.

Stimuli. The stimuli were identical to those used in Group 1 sessions.

Responses. Subjects were trained to discriminate between circles of diameters $8.33 \mathrm{~mm}$ ("small"), $9.398 \mathrm{~mm}$ ("medium"), and $10.32 \mathrm{~mm}$ ("large"), presented for a constant $50 \mathrm{msec}$ and between blank flashes of $30 \mathrm{msec}\left(T_{1}\right)$ and $70 \mathrm{msec}\left(T_{2}\right)$. Subjects were asked on each trial to categorize the size of the circle ("small," "medium," or "large"). At the end of a block of 24 trials, subjects gave judgments as to the frequency of $T_{1}$ and $T_{2}$. After receiving training in the discriminations and before beginning the experimental blocks, subjects were given a practice block of eight trials without feedback.

Procedure. Each subject received five experimental blocks, each consisting of 24 trials. The procedure of trial presentation was identical to that used in Group 1 sessions. The five blocks included the following combinations of stimulus attribute frequencies: $\left(\mathrm{p}_{\mathrm{a}}, \mathrm{p}_{\mathrm{t}}\right)=(1 / 3,1 / 2),(2 / 3,1 / 2),(1 / 2,1 / 2),(1 / 2,1 / 3)$, $(1 / 2,2 / 3)$. The order of presentation of blocks was counterbalanced as for Group 1, and the within-block order of stimuli was randomized.

\section{Group 3: Size and Duration Judgments}

Subjects. Sixteen Stanford University undergraduates received credit toward "Introductory Psychology" for participa- 
tion in individual sessions of about $1 \mathrm{~h}$.

Stimuli. Stimuli used in the time-judgment blocks were a blank flash and the $A_{1}$ circle $(8.33 \mathrm{~mm}$ in diam), shown for 30 and $70 \mathrm{msec}$. Stimuli for the size-judgment blocks were the $A_{1}$ and $A_{2}(10.32 \mathrm{~mm}$ in diam) circles shown for 30 and $70 \mathrm{msec}$.

Responses. Subjects were trained to discriminate between blank flashes of 15, 50, $85 \mathrm{msec}$ (as in Group 1) and between circles of $8.33,9.39$, and $10.32 \mathrm{~mm}$ in diam shown for $50 \mathrm{msec}$ (as in Group 2). During the time judgment blocks, subjects were asked to respond "short," "medium," or "long" to presentations of each stimulus. At the end of these blocks, subjects gave judgments as to the frequency of blanks and circles $\left(A_{1}\right)$ in the block of 24 trials. During the size-judgment blocks, subjects were asked to respond "small," "medium," or "large" to the stimuli and to estimate the relative frequency of $T_{1}$ and $T_{2}$ at the end of each block. In addition to training at the outset of the session, subjects received four trials of practice before beginning the set of time-judgment blocks and four practice trials before beginning the set of size-judgment blocks.

Procedure. Each subject received five blocks consisting of 24 trials per block, with trial presentation procedure identical to that used for Groups 1 and 2. Three trial blocks required temporal judgments on each trial and consisted of the following set of stimulus attribute frequencies: $\left(p_{a}, p_{t}\right)=(1 / 2,1 / 3)$, $(1 / 2,2 / 3),(1 / 2,1 / 2)$. Two blocks consisted of judgments of the sizes of $A_{1}$ and $A_{2}$ presented for 30 or $70 \mathrm{msec}$ and with the following combinations of frequencies: $\left(p_{a}, p_{t}\right)=(1 / 2,2 / 3)$, $(1 / 2,1 / 3)$. The presentation of trial blocks was counterbalanced over subjects, within the constraint that the three time blocks appear consecutively, and the two size blocks appear consecutively. Within-block stimulus orders were randomized and kept constant over subjects.

\section{RESULTS}

The temporal judgments, "short," "medium," and "long," were coded $0,1,2$, respectively, and, for each subject and trial block $\left[\left(\mathrm{p}_{\mathrm{a}}, \mathrm{p}_{t}\right)\right.$ combination], the average was found for each stimulus type. The size judgments, "small," "medium," and "large," were coded 0 , 1,2 and averaged in the same manner as the temporal judgments. Time and size judgments from the three groups of subjects were subjected to separate, parallel analysis of variance, with stimulus attribute frequency, $P$ (type of trial block), stimulus area, A, and stimulus duration, $\mathrm{T}$, as the within-subject variables of interest. Finally, for each type of judgment, the average response conditional on the previous trial was found.

We first consider the temporal judgments $(\tau)$ from Group 1. Separate analyses of variance were performed on the data from the trial blocks in which $\mathrm{p}_{\mathrm{a}}$ was varied $(1 / 3$ or $2 / 3)$ and in which $p_{t}$ was varied $(1 / 3$ or $2 / 3)$. As in previous studies (Avant et al., 1975; Mo \& Michalski, 1972; Thomas \& Cantor, 1975), in both analyses $\tau$ was found to vary directly with $\mathrm{A}[\mathrm{F}(1,20)=$ $18.43, \mathrm{p}<.001 ; \mathrm{F}(1,20)=38.23, \mathrm{p}<.001]]$ and with $\mathrm{T}[\mathrm{F}(1,20)=164.97, \mathrm{p}<.001 ; \mathrm{F}(1,20)=205.12$, $\mathrm{p}<.001]$. For the two blocks in which $\mathrm{p}_{\mathrm{t}}$ was different from $1 / 2$, the effect of $A$ on $\tau$ was greater when $T$ was $70 \mathrm{msec}$ than when $\mathrm{T}$ was $30 \mathrm{msec}[\mathrm{F}(1,20)=4.12$, $\mathrm{p}<.10]$. This $\mathrm{A}$ by $\mathrm{T}$ interaction was not obtained when the other two blocks were considered.

Considering now the effects of varying one of $p_{a}$ and $p_{t}$ while fixing the other at $1 / 2$, it was found that $\tau$ increased as $\mathrm{p}_{\mathrm{a}}$ increased $[\mathrm{F}(1,20)=23.89, \mathrm{p}<.001]$ and as $p_{t}$ increased $[F(1,20)=12.28, p<.005]$. This observed effect of $p_{t}$ is consistent with that predicted by Assumption 2 above, but inconsistent with that implied by Assumption 1. The observed effect of $p_{a}$ is the same for "large" as for "small" circles. The models obtained by combining Assumptions 1 and 2, on the one hand, with Assumptions 3 and 4, on the other, all predict that the effect of $\mathrm{p}_{\mathrm{a}}$ on $\tau$ should be different for "large" and "small" circles. Therefore, the data are inconsistent with these "full" models. On pooling the data across blocks, it was found that $\tau$ for 30 - and 70 -msec durations was less if the preceding stimulus duration was $70 \mathrm{msec}$ than if it was $30 \mathrm{msec}$ (.45 vs. .65 and 1.05 vs. 1.16). This result would be expected if the subjective standard $c^{*}$ in Assumption 2 is influenced most by the most recent stimulus. The model proposed by Thomas and Cantor (1975) makes no predictions about sequential effects. The temporal judgment data, averaged over subjects in Group 1, are presented in Figure 2.

Next we consider the size judgments ( $\alpha$ ) of Group 2 . As in Group 1, separate analyses were performed on the data from blocks in which $p_{t}$ was varied while $p_{a}$ remained at $1 / 2$ and in which $\mathrm{p}_{\mathrm{a}}$ was varied while $\mathrm{p}_{t}$

\begin{tabular}{c|c|c|c}
\multicolumn{2}{c}{$\begin{array}{c}\text { STIMULUS ATTRIBUTE } \\
\text { FREQUENCY }\end{array}$} & \multicolumn{2}{l}{ DURATION } \\
\hline$P_{0}$ & $P_{1}$ & 30msec & 70msec \\
\hline .5 & .7 & 0 & 0 \\
.5 & .3 & 0 & 0 \\
.5 & .5 & 0 & 0 \\
.7 & .5 & 0 & 0 \\
.3 & .5 & 0 & 0 \\
\hline
\end{tabular}

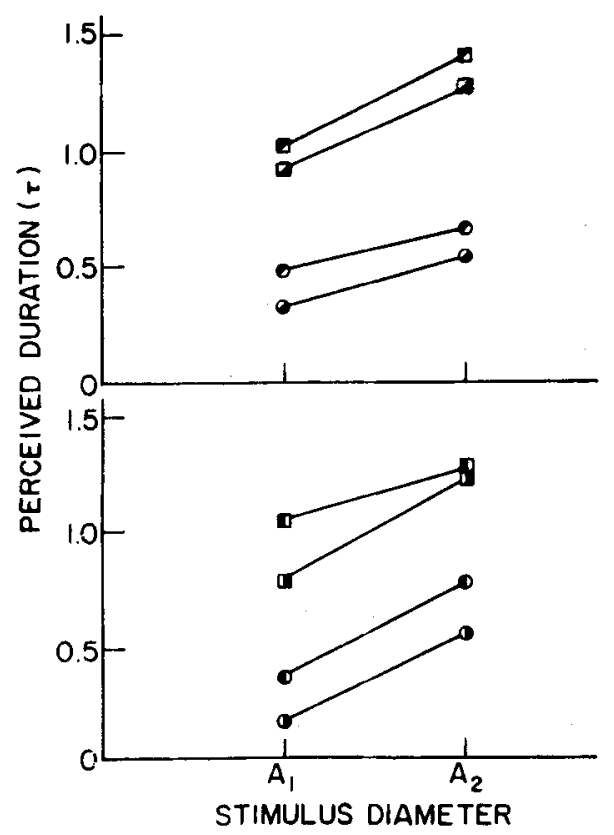

Figure 2. Temporal judgments, averaged over subjects, for each stimulus duration and stimulus area, as attribute frequency is varied; Group 1. 


\begin{tabular}{c|c|c|c}
\multicolumn{3}{c|}{$\begin{array}{l}\text { STIMULUS AT TRIBUTE } \\
\text { FREQUENCY }\end{array}$} & \multicolumn{2}{l}{ DURATION } \\
\hline$P_{0}$ & $p_{1}$ & $30 \mathrm{msec}$ & $70 \mathrm{msec}$ \\
\hline .5 & .7 & 0 & 0 \\
.5 & .3 & 0 & 0 \\
.5 & 5 & 0 & 0 \\
7 & .5 & 0 & 0 \\
.3 & 5 & 0 & 0 \\
\hline
\end{tabular}

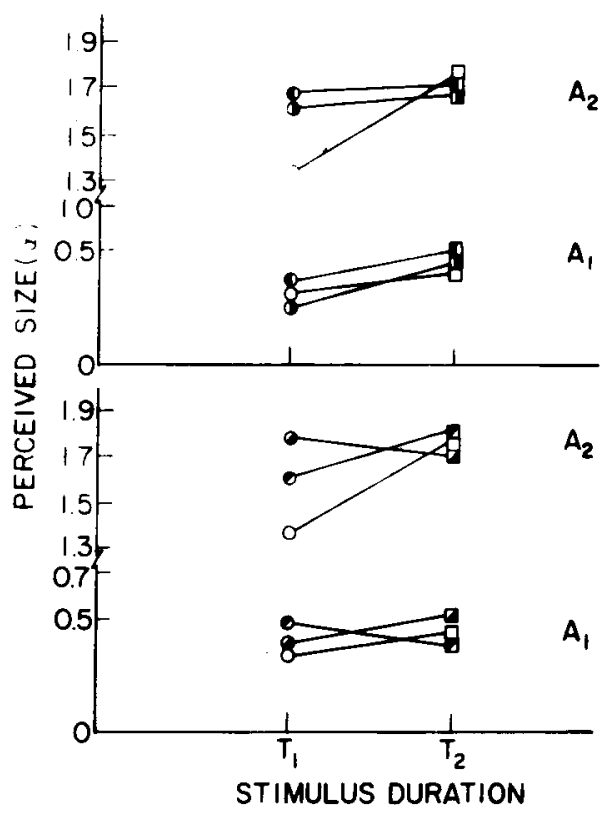

Figure 3. Size judgments, averaged over subjects, for each stimulus duration and stimulus area, as attribute frequency $\left(\mathrm{p}_{\mathrm{a}}, \mathrm{p}_{\mathrm{t}}\right)$, is varied; Group 2.

remained at $1 / 2$. Each analysis compared the judgments from the trial blocks in which frequency was varied to that in which $\mathrm{p}_{\mathrm{a}}$ and $\mathrm{p}_{\mathrm{t}}$ were set at $1 / 2$. As in a previous study (Thomas \& Cantor, 1975), $\alpha$, when averaged across blocks, was found to vary directly with $T$ for both values of $A$ in both analyses $[F(1,20)=11.04, p<.005$; $F(1,20)=31.76, p<.001]$. As with Group 1 subjects, when considering blocks in which $p_{t}$ was varied, the effect of $T$ on $\alpha$ was greater for "large" circles than for "small" circles $[F(1,20)=6.05, p<.025]$. There are, of course, significant main effects due to $A$ in both analyses.

Considering now the effects of varying one of $p_{a}$ and $p_{t}$ while fixing the other at $1 / 2$, it was found that varying $p_{t}$ and varying $p_{a}$ both produced significant changes in $\alpha[F(2,40)=3.29, p<.05 ; F(2,40)=3.8$, $p<.05$, respectively]. However, the frequency effect is not monotonic, inasmuch as $\alpha$ is smallest in the $(1 / 2,1 / 2)$ block because of the underestimation of large circles exposed for $30 \mathrm{msec}$ in this block. The small average for this stimulus contributes to a significant $P$ by $T$ interaction, when $p_{t}$ is varied, and significant $P$ by $A$ by $T$ interactions in both analyses. It should be noted that $\alpha$ is approximately the same when $p_{t}=1 / 3$ as when $p_{t}=2 / 3$, but is less, for all stimuli, when $p_{a}=$ $1 / 3$ than when $\mathrm{p}_{\mathrm{a}}=2 / 3$. This difference in $\alpha$ due to $\mathrm{p}_{\mathrm{a}}$ is consistent with Assumption 2 but not with Assumption 1. Finally, the direct effect of $\mathrm{T}$ on $\alpha$ holds for all stimuli and $\left(\mathrm{p}_{\mathrm{a}}, \mathrm{p}_{\mathrm{t}}\right)$ combinations, with the exception of "large" circles when $p_{t}=1 / 3$ and "small" circles when $p_{t}=2 / 3$. These reversals also contribute to the $P$ by $A$ by $T$ interaction observed when $p_{t}$ was varied. On pooling the data across blocks, it was found that $\alpha$ for $A_{1}$ and for $A_{2}$ was less if the preceding stimulus was $A_{2}$ than if it was $A_{1}$ (.40 vs. .50 and 1.57 vs. 1.72). The size judgment data, averaged over subjects in Group 2, are presented in Figure 3.

The third group of subjects was run to see what effect variation of $\mathrm{p}_{t}$ would have on the temporal judgments of blank intervals. It was decided to also get size judgments of "large" and "small" circles to see if the reversals in the effect of $T$ on $\alpha$, observed in Group 2, would be replicated.

Considering first the size judgments, it was found that $T$ has a direct effect on $\alpha$ for both "large" and "small" circles and both values of $p_{t}, 1 / 3$ and $2 / 3$ $[F(1,15)=8.31, p<.025]$. No interaction was significant. Also, $\alpha$ was significantly less when $p_{t}=1 / 3$ than when $\mathrm{p}_{\mathrm{t}}=2 / 3[\mathrm{~F}(1,15)=7.15, \mathrm{p}<.025]$. Thus

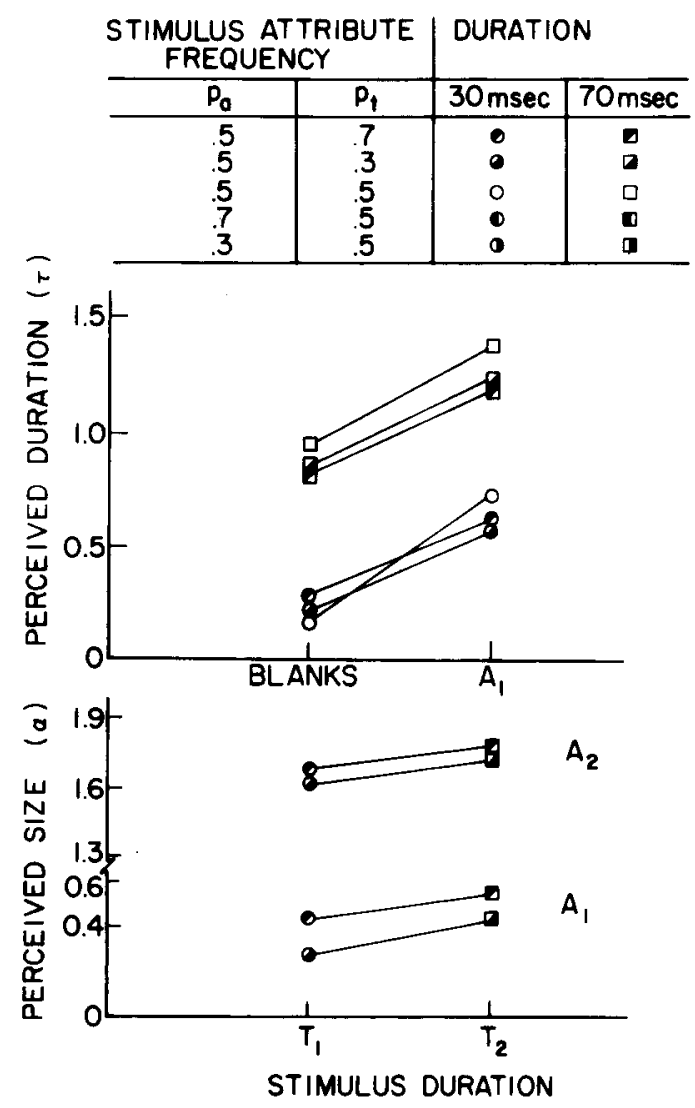

Figure 4. Temporal judgments, averaged over subjects, for each stimulus area (blanks and small circles) and stimulus duration $\left(T_{1}, T_{2}\right)$ and size judgments, averaged over subjects, for each stimulus area $\left(A_{1}, A_{2}\right)$ and stimulus duration $\left(T_{1}, T_{2}\right)$ as attribute frequency $\left(\mathrm{p}_{\mathrm{a}}, \mathrm{p}_{\mathrm{t}}\right)$ is varied; Group 3 . 
the reversals observed in Group 2 were not replicated.

Next we consider the temporal judgments of 30 or 70 -msec durations which were equally likely to be blank or contain a small circle ("filled"). The frequency, $\mathrm{p}_{t}$, took on values $1 / 3,1 / 2$, and $2 / 3$. As expected, $\tau$ was greater for "filled" than for blank durations $[F(1,15)=10.45, p<.01]$, and increased with $T$ $[F(1,15)=76.5, p<.001]$. Varying $p_{t}$ did not have a significant effect on $\tau[F(2,30)=1.5]$, nor was there any significant interaction involving $\mathrm{p}_{t}$. With the exception of 30 -msec blank durations, $\tau$ was greatest when $\mathrm{p}_{t}=1 / 2$, this (nonsignificant) effect holding for 10 of the 16 subjects. The temporal and size judgment data are each presented separately in Figure 4, averaged over subjects in Group 3.

The last analysis of the data consisted of replotting some of the data to show the relationship between $\tau$ and $\alpha$, for each stimulus, as $\left(\mathrm{p}_{\mathbf{a}}, \mathrm{p}_{\mathfrak{t}}\right)$ is varied. As pointed out earlier, the models based on Assumptions 1 to 4 predict a direct relationship for only some stimuli, whereas the model proposed by Thomas and Cantor (1975) provides two ways of accounting for a direct relationship. For each of the four stimuli $\left(A_{i}, T_{j}\right)$ and each of four values of $\left(p_{a}, p_{t}\right),(1 / 3,1 / 2),(2 / 3,1 / 2),(1 / 2,1 / 3)$, and $(1 / 2,2 / 3)$, the values of $\alpha$ obtained from Groups 2 and 3 were plotted against the value of $\tau$ obtained from Group 1. It can be seen that, for each stimulus, the relationship between $\alpha$ and $\tau$ is generally a direct one. The four clusters of points fall at the vertices of a "parallelogram" (the broken lines in Figure 5), showing the direct relationship between $\alpha$ and $T$ (for each value of $A$ ) and between $\tau$ and $A$ (for each value of $T$ ). This analysis of the temporal and size judgments of the three subject groups is presented in Figure 5.

\section{DISCUSSION}

To summarize the main findings, data from Groups 1 and 3 suggest that perceived duration varies directly with amount of presented visual information for all values of presented duration and attribute frequencies $\left(p_{a}, p_{t}\right)$ under consideration. Furthermore, perceived size is seen to vary directly with presented duration, when presented size frequency has the values $p_{a}=1 / 3$ and $2 / 3$ (Group 2), and when temporal frequency has the values $p_{t}=1 / 3$ and $2 / 3$ (Group 3 ). These results are consistent with those of Thomas and Cantor (1975), who set $p_{a}=p_{t}=1 / 2$. The manipulations of $p_{a}$ and $p_{t}$ lead to similar effects on both perceived size and perceived duration. For each stimulus, perceived duration is seen to vary directly with both $\mathrm{p}_{\mathrm{a}}$ (frequency of small circles) and $p_{t}$ (frequency of short duration) (Groups 1 and 3); and perceived size shows a similar dependence on $\mathrm{p}_{\mathrm{a}}$ and $\mathrm{p}_{t}$ (Groups 2 and 3). Comparing data across the three groups, it can be seen that perceived duration and perceived size vary directly with each other under changes in stimulus attribute frequency. However, when temporal judgments of blank and "filled" intervals are required, perceived

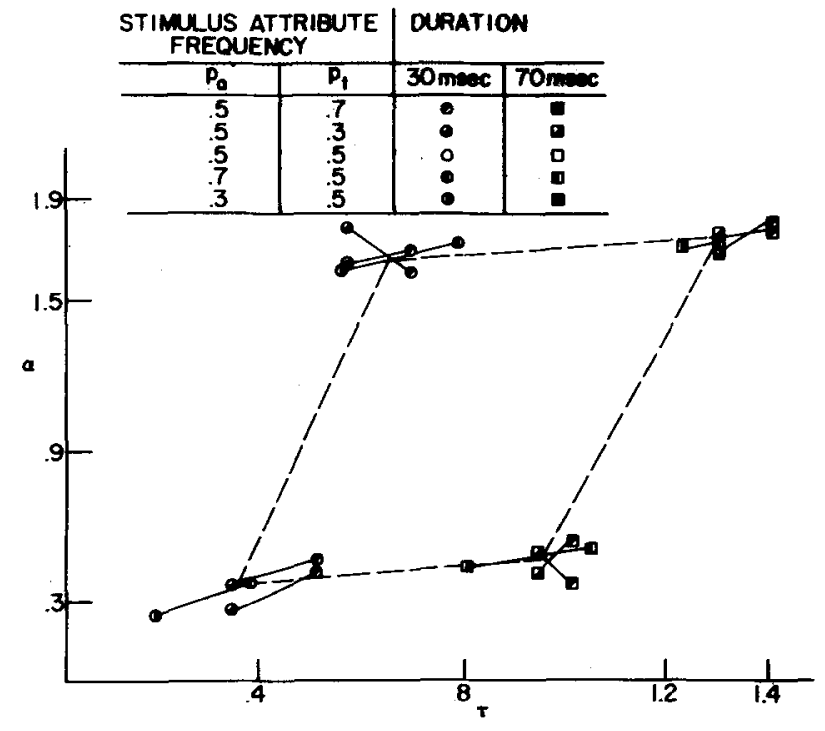

Figure 5. Perceived duration $(\tau)$ vs. perceived size $(\alpha)$ for each stimulus as attribute frequency $\left(\mathrm{p}_{\mathrm{a}}, \mathrm{p}_{t}\right)$ is varied. The solid lines connect pairs of points with different $p_{a}$ or $p_{t}$ values for which $\tau$ comes from Group 1, and $\alpha$ comes from Group 2 or from Group 3. The dashed lines connect points which are the mean values of $\alpha$ and $\tau$, averaged across $\mathrm{p}_{\mathrm{a} t}$ values for each stimulus.

duration is no longer affected by changes in $\mathbf{p}_{t}$ (Group 3).

The results of the present study can be accounted for by the model proposed by Thomas and Cantor (1975) in which perceived duration is assumed to be a function both of presented duration and time spent processing presented visual information. The sampling mechanism proposed in such a model allows one to predict the direct relation found between $\alpha$ and $\tau$ as a result of the growth of both apparent size and apparent duration over PT. This is because variations in stimulus attribute frequency are expected to affect PT by changing the rate of growth of apparent size over time, or by changing the criterion of sample "quality" at which sampling stops. Changes in the rate of growth could occur because the subject uses a systematic rather than a random sampling strategy, or because the subject changes the rate at which samples are taken. This expected effect on PT will occur provided the task requires a significant amount of processing of size information, as in Groups 1 and 2. However, the task required of the third group of subjects does not require much processing of size information simply because the alternative stimuli were small circles or blank durations. Therefore, we would no longer expect an effect of stimulus attribute frequency on $\tau$. Indeed, for this group of subjects, variations in $p_{t}$ has no effect on $\tau$.

Models such as those obtained by combining Assumptions 1 to 4 cannot account for the parallel increases, obtained in this study for all stimuli, in both $\alpha$ and $\tau$ with increases in either $p_{a}$ or $p_{t}$. Further, the modification of Assumption 2, which makes the standard $c^{*}$ most sensitive to the most recent stimulus, can account for only part of the data, namely, the effects of $\mathrm{p}_{\mathrm{a}}$ on 
$\alpha, \mathrm{p}_{\mathrm{t}}$ on $\tau$ [because, in this experiment, the probability that the preceding stimulus is $T_{1}$ (or $A_{1}$ ) increases with $\mathrm{p}_{\mathrm{t}}\left(\right.$ or $\left.\mathrm{p}_{\mathrm{a}}\right)$ ] and, of course, the observed sequential effects. Similarly, models that attempt to account for the data in terms of response biases (for example, a tendency to use each response alternative equally often), make no predictions about the effects of stimulus area on perceived duration and of stimulus duration on perceived size.

Yet another model of time perception is the storage size theory (Ornstein, 1969). A naive version of this theory might suggest that, for any trial block, $\tau$ is directly related to the average presented size. By such a theory, $\tau$ would be expected to decrease as $\mathrm{p}_{\mathrm{a}}$ increased, which is contrary to the present data. A more detailed application of the storage size hypothesis might simply assert that $\tau$ is directly related to $\alpha$ rather than to $\mathbf{A}_{\mathbf{i}}$. However, such an explanation would not help to explicate the reasons for the specific changes in $\alpha$ and $\tau$ found with certain manipulations, such as varying stimulus attribute frequency. In contrast, the assumption of a sampling mechanism, the rate and the operating time of which might be varied, allows for simultaneous changes in the size of the encoding of useful visual information (of which $\alpha$ is a function) and in the processing time (of which $\tau$ is a function).

The direct relation between $\tau$ and $\alpha$ is mediated through changes in the time spent processing useful visual information. A technique that appears to control successfully amount of visual processing is that of using a visual mask (Moore \& Massaro, 1973). This technique has been applied to the present context in a very recent study by Cantor and Thomas (1976).

\section{REFERENCES}

Allan. L. G.. Kristofferson. A. B., \& Wiens, E. W. Duration discrimination of brief light flashes. Perception \& Psychophysics, 1971, 9. 327-334.
Avant, L. L., Lyman, P. J., \& Antes, J. R. Effects of stimulus familiarity on judged visual duration. Perception \& Psychophysics, 1975, 17, 253.262.

Cantor, N. E., \& Thomas, E. A. C. Visual masking effects on duration, size, and form discrimination. Perception \& Psychophysics, 1976, 19, 321-327.

Green, D. M., \& Swets, J. A. Signal detection theory and psychophysics. New York: Wiley, 1966.

Helson, H. Adaptation-level as a frame of reference for prediction of psychological data. American Journal of Psychology, 1947, 60, 1-69.

LAMNG, D. R. J. Information theory of choice-reaction times. New York: Academic Press, 1968.

Link. S. W., \& HEATH, R. A. A theory of sequential comparative judgment. Psychometrika, 1975, 40, 77-106.

Mo, S. S. Temporal reproduction of duration as a function of numerosity. Bulletin of the Psychonomic Society, 1975, 5. $165-167$.

Mo, S. S., \& Michalski, V. A. Judgments of temporal duration of area as a function of stimulus configuration. Psychonomic Science, 1972, 27, 97-98.

Moore, J. J., \& Massaro, D. W. Attention and processing capacity in auditory recognition. Journal of Experimental Psychology, 1973, 99, 49-54.

ORNSTEIN, R. On the experience of time. Baltimore, Penguin, 1969.

Thомаs, E. A. C. On expectancy and the speed and accuracy of responses. In $\mathrm{S}$. Kornblum (Ed.), Attention and performance IV. New York: Academic Press, 1973.

Thomas, E. A. C., \& Cantor, N. E. On the duality of simultaneous time and size perception. Perception \& Psychophysics, 1975, 18, 44.48.

Thomas, E. A. C., \& Myers, J. L. Implications of latency data for threshold and nonthreshold models of signal detection. Journal of Mathematical Psychology, 1972, 9. 253-285.

Thomas, E. A. C., \& Weaver, W. B. Cognitive processing and time perception. Perception \& Psychophysics, 1975, 17. 363-367.
(Received for publication September 10, 1975: revision accepted January $7,1976$. ) 